\title{
LA PARTICIPACIÓN POLÍTICA DE LAS MUJERES EN EUSKADI: DATOS Y REFLEXIONES
}

\author{
ARANTXA ELIZONDO LOPETEGI \\ Universidad del País Vasco
}

\section{LA PARTICIPACIÓN POLÍTICA: PRECISIONES TEÓRICAS}

¿Qué se entiende por participación política? ¿Cómo se manifiesta esa participación? ¿Cuáles son las principales modalidades de participación en una sociedad concreta? ¿Quiénes participan o se implican más en política? Estas son algunas de las preguntas más frecuentes en la Ciencia Política contemporánea. Cualquiera que se detenga siquiera someramente a revisar las numerosas investigaciones sobre la materia llegará inevitablemente a la conclusión de que la participación política no tiene una definición clara y consensuada. La amplia producción empírica genera una gran variedad conceptual puesto que la participación política se distingue por ser casi siempre un concepto relativo y aplicado que se ajusta y delimita a la investigación concreta de que se trate.

No obstante, el concepto teórico en el que se sustenta la noción de participación hace referencia al contenido esencial de la propia definición de democracia como «el poder del pueblo». De esta esencia abstracta se deriva el funcionamiento democrático en la práctica como un sistema de control entre poderes ubicado en un escenario en el que participan diversos actores sociales, tanto individuales como colectivos. En este sentido, la participación política significa tomar parte o intervenir en la gestión de lo colectivo, de lo que afecta a la sociedad en su conjunto. Participar no es tan sólo colaborar ni opinar, sino que supone la voluntad o el sentimiento de pertenencia a un colectivo, grupo o comunidad ${ }^{1}$.

La participación política se manifiesta en diversas modalidades o formas que incluyen distintas dimensiones: canales institucionales como votar en las elecciones, presentarse en una candidatura, formar parte de una comisión, etc.; la pertenencia a los principales actores que tradicionalmente han sido prota-

1. PINDADO, Fernando: La participación ciudadana en la vida de las ciudades, Barcelona, Ediciones del Serbal, 2000, p. 21. 
gonistas en la sociedad (partidos, sindicatos) y a otro tipo de organizaciones o asociaciones; y el desarrollo de actos individuales o colectivos dirigidos a influir directa o indirectamente sobre la toma de decisiones de los poderes públicos. Durante las últimas décadas, además, hemos asistido al incremento de los procedimientos de participación ciudadana orientados fundamentalmente a abrir foros de deliberación y debate tanto para actores organizados como para la ciudadanía no organizada.

La variedad en las modalidades de participación se corresponde con la gran diversidad en los niveles de implicación que conlleva cada una de ellas. Los individuos participan de forma desigual en el ejercicio de influencia política por distintas razones, aunque se puede considerar que esta desigualdad refleja a su vez las diferencias sociales y económicas existentes entre los miembros de la sociedad. Las personas no están igualmente provistas de recursos (cognitivos, disposición de tiempo, habilidades personales, relaciones...) lo cual incide directamente en su actividad política ${ }^{2}$. En este sentido, muchas investigaciones pretenden definir el perfil y las características concretas de quienes participan en política elaborando categorías en función del grado de participación. Por lo general, se ha encontrado relación entre la participación y variables como la edad, el nivel de instrucción, la posición profesional o el sexo.

El inicio del estudio sistemático de las diferencias en las pautas de participación política en función de la variable sexo debe entenderse en el contexto del auge del behaviorismo imperante en la década de los cincuenta. Desde entonces ha habido una clara evolución en las perspectivas analíticas ${ }^{3}$ : según el modelo utilizado hasta los años setenta, el sexo constituía una de las principales variables explicativas de la actividad política junto a la edad, la educación y la clase social. En esta época el concepto de participación política se limitaba a la afiliación a organizaciones políticas formales (partidos y sindicatos), la ocupación de cargos políticos e institucionales, la actividad en las campañas electorales y el voto. Teniendo en cuenta estas esferas, los hombres eran políticamente más activos que las mujeres. Las principales explicaciones a estas tasas de mayor actividad masculina apuntaban fundamentalmente a las diferencias en el proceso de socialización de hombres y mujeres ${ }^{4}$.

A partir de los años ochenta las nuevas teorías de la participación política, en las que el feminismo tendrá un importante impacto, critican que hasta ese momento la política ha sido entendida como la actividad de las instituciones

2. Verba, Sidney, Norman H. Nie y Jae-on Kim: Participation and Political Equality: A Seven-Nation Comparison, Chicago, University of Chicago Press, 1978, p. 1.

3. NorRIS, Pippa: "Gender Differences in Political Participation in Britain: Traditional, Radical and Revisionist Models», Government and Opposition, 26-1 (1991), pp. 56-74. Una explicación detallada de esta evolución puede encontrarse en ELIzONDO, Arantxa: La presencia de las mujeres en los partidos politicos de la CAPV, Vitoria, Gobierno Vasco, 1999, pp. 43-50.

4. Easton, David y Dennis, Jack: Children and the Political System, Chicago, Chicago University Press, 1980 (edición original de 1969), p. 336; IGLITzin, Lynne: "The Making of the Apolitical Woman: Feminity and Sex-Stereotyping in Girls», en Jane S. Jaquette (ed): Women in Politics, Nueva York, John Wiley, 1974, p. 31. 
y la participación política se ha limitado a considerar la afiliación a organizaciones políticas tradicionales, el ejercicio del voto y la ocupación de cargos de representación. Exigen que el concepto sea entendido de una manera mucho más amplia, incluyendo las nuevas formas de actividad en las que las mujeres tienen una presencia superior a la observada en las formas convencionales ${ }^{5}$. Esta perspectiva afirma que la propia Ciencia Política deforma la percepción de las diferencias basadas en el sexo porque los parámetros de medida han sido masculinos. En consecuencia, las investigaciones anteriores han omitido el papel de las mujeres en la política tan sólo porque se ha desarrollado en un escenario invisible para los autores e irrelevante para la investigación politológica.

Durante la misma época, algunas investigaciones se dedican a revisar los resultados obtenidos en las décadas anteriores. Reconociendo la influencia de la variable sexo, consideran que se han exagerado las diferencias. Este enfoque subraya la necesidad de romper con los estereotipos sexuales delatando el supuesto implícito en los estudios anteriores, según el cual los sexos constituyen dos grupos contrapuestos entre sí. Además, señala que en los últimos años las diferencias de sexo empiezan a remitir y el comportamiento político de hombres y mujeres se va asemejando progresivamente ${ }^{6}$. Según esta perspectiva, los cambios sociales producidos en la forma de vida de las mujeres (destacando el aumento de los niveles de educación y el acceso al mercado laboral) conllevarán a largo plazo una equiparación de sus actividades políticas con las de los hombres?.

En suma, según la práctica totalidad de las investigaciones el sexo sigue siendo una variable fundamental relacionada con la actividad política: los hombres son más activos, rasgo que se repite en prácticamente todos los tipos de participación política ${ }^{8}$. De hecho, los estudios empíricos consideran el sexo como uno de los más significativos predictores para la participación. Podemos afirmar que la discriminación de las mujeres en distintos ámbitos sociales y económicos y la dedicación a las actividades que tradicionalmente se les han asignado han impedido o, en el mejor de los casos, obstaculizado su participación en la vida política.

¿Sigue siendo esto válido para las sociedades actuales? ¿Cómo se refleja el acceso de las mujeres a ámbitos políticos que les estaban vedados hasta hace algunas décadas? El objetivo de este artículo consiste en retratar esta desigualdad en la participación política de mujeres y hombres en el caso de la Comunidad Autónoma del País Vasco (en adelante CAPV). Más allá de la descripción concreta, los datos pretenden servir como punto de partida para la reflexión acerca

5. Randall, Vicky: Women and Politics: An International Perspective, Londres, MacMillan, 1987, p. 59.

6. BескWIтH, Karen: American women and political participation, Westport, Greenwood Press, 1986, p. 31.

7. Norris, Pippa: «Gender Differences...», op. cit., p. 63.

8. Verba, Sidney, Norman H. Nie y Jae-on Kim: Op. cit., p. 234; Dalton, Russell J.: Citizen Politics, New Jersey, Chatham, 1996, p. 55; Norris, Pippa: Critical Citizens, Oxford, Oxford University Press, 1999, p. 261; Duverger, Maurice: The Political Role of Women, Nueva York, UNESCO, 1955. 
de cuestiones como la importancia de la participación en el desarrollo teórico de la democracia, el reparto desigual de recursos políticos en la sociedad, el debate acerca de la necesidad de empoderar a las mujeres y, unido a esto, la conveniencia de establecer medidas concretas orientadas a incrementar su participación política.

El concepto de participación política utilizado en este artículo toma como punto de partida la siguiente clasificación:

1) Participación institucional

2) Participación organizada

3) Participación individual

La información compilada a lo largo del artículo se limita a ofrecer una serie de indicadores referidos a cada una de las tres dimensiones y provenientes, en su mayor parte, de los Informes de Cifras sobre la Situación de Mujeres y Hombres en Euskadi que el Instituto Vasco de la Mujer-Emakunde elabora con periodicidad anual ${ }^{9}$. La participación institucional engloba datos acerca de la presencia de mujeres tanto en puestos políticos de representación como en cargos ejecutivos en los diferentes niveles institucionales (autonómico, foral y municipal). La participación organizada incluye información acerca de la proporción de mujeres en los partidos políticos y sindicatos así como su pertenencia a diversas organizaciones y asociaciones. Por último, la participación individual recoge aspectos más relacionados con la actitud general hacia la política que con la participación propiamente dicha. Así, tiene en cuenta las posibles diferencias en función del sexo en el interés por la política y la disposición a tomar parte en actividades concretas.

\section{PRESENCIA DE MUJERES EN LAS INSTITUCIONES VASCAS}

A pesar del incremento experimentado en las últimas décadas, la presencia de mujeres en las instituciones de representación política y en los cargos políticos y de la administración en la CAPV sigue siendo aún muy inferior a la de los

Tabla 1. Evolución del porcentaje de mujeres y hombres en el Parlamento Vasco, 1980-2001

\begin{tabular}{|c|r|r|r|r|r|r|r|}
\hline & $\mathbf{1 9 8 0}$ & $\mathbf{1 9 8 4}$ & $\mathbf{1 9 8 6}$ & $\mathbf{1 9 9 0}$ & $\mathbf{1 9 9 4}$ & $\mathbf{1 9 9 8}$ & $\mathbf{2 0 0 1}$ \\
\hline Mujeres & 4 & 8 & 10 & 15 & 18 & 22 & 26 \\
$\mathbf{\%}$ & 6,7 & 10,7 & 13,3 & 20 & 24 & 29,3 & 34,7 \\
\hline Hombres & 56 & 67 & 65 & 60 & 57 & 53 & 49 \\
\% & 93,3 & 89,3 & 86,7 & 80 & 76 & 70,7 & 65,3 \\
\hline Total & 60 & 75 & 75 & 75 & 75 & 75 & 75 \\
\hline
\end{tabular}

Fuente: Emakunde: Cifras sobre la Situación de Mujeres y Hombres en Euskadi 2001.

9. La autora de este artículo ha coordinado el grupo de trabajo responsable de la realización de dicho informe anual en las ediciones de 2001, 2002 y 2003 e integrado por Eva Martínez Hernández, Ainhoa Novo Arbona y Raquel Sanz Álvarez, profesoras e investigadoras del Departamento de Ciencia Política y la Administración de la Universidad del País Vasco. 
Tabla 2. Altos cargos en el Gobierno Vasco según el sexo, 2002

\begin{tabular}{|l|c|r|r|r|r|}
\hline & Total & \multicolumn{2}{|c|}{ Mujeres } & \multicolumn{2}{c|}{ Hombres } \\
\cline { 2 - 6 } & Abs. & Abs. & \% & Abs. & \% \\
\hline Consejerías & 11 & 3 & 27,3 & 8 & 72,7 \\
\hline Viceconsejerías & 35 & 2 & 5,7 & 33 & 94,3 \\
\hline Direcciones & 120 & 29 & 24,2 & 91 & 75,8 \\
\hline Total & 166 & 34 & 20,5 & 132 & 79,5 \\
\hline
\end{tabular}

Fuente: Emakunde: Cifras sobre la Situación de Mujeres y Hombres en Euskadi 2002.

hombres. En el ámbito autonómico, las mujeres ocupan un tercio de los escaños del Parlamento Vasco actual y representan una quinta parte de los altos cargos del Gobierno Vasco. A pesar de que la situación se aleja mucho del 6,7\% de mujeres parlamentarias de 1980, es llamativo el hecho de que el nivel ejecutivo siga siendo más cerrado al acceso de las mujeres: sólo el $20 \%$ de los altos cargos del Gobierno Vasco (teniendo en cuenta el conjunto de las personas responsables de consejerías, viceconsejerías y direcciones) son ocupados por mujeres.

La literatura sobre la presencia femenina en las instituciones suele insistir en la idea de que en algunas áreas institucionales el acceso de las mujeres ha sido más visible y destaca que esferas más relacionadas con el rol que han ejercido históricamente, como Asuntos Sociales, Cultura, Sanidad o Educación, son más permeables a la actividad de las mujeres políticas. En nuestro caso, si tenemos en cuenta los datos de la presencia de mujeres en cargos directivos dentro de cada departamento, observamos que en Cultura se acerca a la mitad (el 45,5\%); en el otro extremo, la presencia es nula en Agricultura y Pesca y, contraria-

Tabla 3. Porcentaje de mujeres y hombres en altos cargos (viceconsejerías y direcciones) en el Gobierno Vasco según Departamentos, 2002

\begin{tabular}{|l|c|c|}
\hline & Mujeres $\%$ & Hombres $\%$ \\
\hline Presidencia de gobierno & 18,2 & 81,8 \\
\hline Vicepresidencia de Gobierno & 27,3 & 72,7 \\
\hline Hacienda y Administración Pública & 17,6 & 82,4 \\
\hline Educación Universidades e Investigación & 26,7 & 73,3 \\
\hline Interior & 28,6 & 71,4 \\
\hline Industria, Comercio y Turismo & 17,6 & 82,4 \\
\hline Vivienda y Asuntos Sociales & 10,0 & 90,0 \\
\hline Justicia, Empleo y Seguridad Social & 26,7 & 73,3 \\
\hline Sanidad & 10,0 & 90,0 \\
\hline Cultura & 45,5 & 54,5 \\
\hline Ordenación del Territorio y Medio Ambiente & 12,5 & 87,5 \\
\hline Transporte y Obras Públicas & 16,7 & 83,3 \\
\hline Agricultura y Pesca & 0,0 & 100,0 \\
\hline Total & 20,6 & 79,4 \\
\hline
\end{tabular}

Fuente: Emakunde: Cifras sobre la Situación de Mujeres y Hombres en Euskadi 2002. 
mente a lo que señalan los resultados de numerosas investigaciones, la menor proporción corresponde a las Consejerías de Vivienda y Asuntos Sociales y la de Sanidad.

La presencia de mujeres ha ido en aumento, asimismo, en las Juntas Generales (órganos de representación en los tres Territorios Históricos que integran la CAPV) y en los ayuntamientos. En la actualidad las mujeres ocupan el 30,1\% de los escaños de las Juntas y el $28,4 \%$ de las concejalías de la comunidad. De modo similar al del nivel autonómico, estas cifras se alejan considerablemente de los porcentajes correspondientes a la dimensión ejecutiva. Por ejemplo, en el caso de los ayuntamientos, sólo el 14,3\% de las alcaldías están en manos de una mujer.

Finalmente, la mención a la participación institucional tiene que incluir inevitablemente la referencia a las nuevas estrategias para la implicación, participación y consenso de la ciudadanía en el desarrollo de políticas públicas. La puesta

Tabla 4. Evolución del porcentaje de mujeres en las Juntas Generales de los tres Territorios Históricos, 1979-2003

\begin{tabular}{|l|c|c|c|c|c|c|c|}
\hline & $\mathbf{1 9 7 9}$ & $\mathbf{1 9 8 3}$ & $\mathbf{1 9 8 7}$ & $\mathbf{1 9 9 1}$ & $\mathbf{1 9 9 5}$ & $\mathbf{1 9 9 9}$ & $\mathbf{2 0 0 3}$ \\
\hline Araba & 10,5 & 1,9 & 13,7 & 15,7 & 13,7 & 15,1 & 25,5 \\
\hline Bizkaia & 4,4 & 7,8 & 7,8 & 13,7 & 21,6 & 33,3 & 41,2 \\
\hline Gipuzkoa & 4,9 & 3,9 & 3,9 & 9,8 & 15,7 & 29,4 & 25,5 \\
\hline Total & 6,1 & 4,6 & 4,6 & 13,1 & 17 & 26,5 & 30,1 \\
\hline
\end{tabular}

Fuente: Emakunde: Cifras sobre la Situación de Mujeres y Hombres en Euskadi 2002.

Tabla 5. Evolución de la cantidad de mujeres concejalas en los Ayuntamientos de la CAPV, 1979-1999

\begin{tabular}{|l|r|r|r|r|r|r|r|}
\hline & $\mathbf{1 9 7 9}$ & $\mathbf{1 9 8 3}$ & $\mathbf{1 9 8 7}$ & $\mathbf{1 9 9 1}$ & $\mathbf{1 9 9 5}$ & $\mathbf{1 9 9 9}$ & \multicolumn{1}{c|}{$\mathbf{2 0 0 3}$} \\
\hline Total & $\mathbf{2 . 4 2 8}$ & $\mathbf{2 . 4 5 2}$ & $\mathbf{2 . 5 0 5}$ & $\mathbf{2 . 5 3 9}$ & $\mathbf{2 . 2 6 2}$ & $\mathbf{2 . 5 4 0}$ & $\mathbf{2 . 5 1 1}$ \\
\hline Mujeres & 170 & 201 & 295 & 407 & 512 & 690 & 713 \\
\hline$\%$ & 7,0 & 8,2 & 11,8 & 16,0 & 22,6 & 27,2 & 28,4 \\
\hline
\end{tabular}

Fuente: Emakunde: Cifras sobre la Situación de Mujeres y Hombres en Euskadi 2002.

Tabla 6. Corporaciones y puestos de alcaldía según sexo y año

\begin{tabular}{|l|c|c|c|c|c|}
\hline & Mujeres & \% & Hombres & \% & $\begin{array}{c}\text { Total } \\
\text { municipios }\end{array}$ \\
\hline $\mathbf{1 9 9 1}$ & 23 & 9,3 & 225 & 90,7 & 248 \\
\hline $\mathbf{1 9 9 5}$ & 22 & 8,8 & 228 & 91,2 & 250 \\
\hline $\mathbf{1 9 9 9}$ & 30 & 12,0 & 220 & 88,0 & 250 \\
\hline $\mathbf{2 0 0 3}$ & 35 & 14,3 & 210 & 85,7 & 245 \\
\hline
\end{tabular}

Fuente: Emakunde: Cifras sobre la Situación de Mujeres y Hombres en Euskadi 2002. 
Tabla 7. Ayuntamientos con foro participativo sobre igualdad entre los sexos. 2002

\begin{tabular}{|l|l|}
\hline GIPUZKOA & $\begin{array}{l}\text { Donostia-San Sebastián } \\
\text { Arrasate-Mondragón } \\
\end{array}$ \\
& Eibar \\
& Hernani \\
\hline BIZKAIA & Barakaldo \\
& Basauri \\
& Ermua \\
& Getxo \\
& Ondarroa \\
\hline ARABA & Vitoria-Gasteiz \\
\hline
\end{tabular}

Fuente: Martínez Hernández, Eva: «Participación Ciudadana y Empoderamiento de las Mujeres: Los Consejos de Igualdad en la Comunidad Autónoma del País Vasco", Comunicación presentada en el VI Congreso de la AECPA, Barcelona, 18-20 de septiembre de 2003.

en práctica de estas estrategias se materializa principalmente en la creación de foros para canalizar la consulta y participación ciudadana en la toma de decisiones de la administración. Precisamente, en el caso de las políticas de igualdad, la participación y el empoderamiento de las mujeres es con frecuencia un objetivo en sí mismo. Por eso, la creación de foros de participación constituye un elemento de suma relevancia en materia de igualdad entre mujeres y hombres. En el nivel autonómico de la CAPV existe la Comisión Consultiva del Instituto Vasco de la Mujer-Emakunde que promueve la interrelación del movimiento asociativo de mujeres de los tres territorios históricos con los poderes públi$\cos ^{10}$; en el nivel provincial o foral funcionan foros de participación de mujeres adscritos a la Diputación de Bizkaia y a la Diputación de Araba ${ }^{11}$; y por último, existen en la actualidad 10 foros municipales de este tipo.

\section{MUJERES EN ORGANIZACIONES SOCIALES Y POLÍTICAS}

Los partidos políticos y los sindicatos constituyen dos dimensiones fundamentales en los procesos de participación política en las democracias actuales. El acceso de las mujeres a los ámbitos de toma de decisión está estrechamente relacionado con el papel que tienen en estas instancias. Los datos que muestran al respecto las ejecutivas de los partidos de la CAPV muestran notables diferencias entre las formaciones: el PSE-EE presenta el porcentaje más equilibrado, el 45,5\%, mientras que en el otro extremo destaca el PNV, en cuya ejecutiva sólo hay una mujer sobre 13 , lo que supone un $7,1 \%$ sobre el total. Por lo que respecta a los principales sindicatos de la CAPV, la presencia de mujeres en las ejecutiva es en general inferior a la de los partidos. Así, los porcentajes más

10. La Comisión está regulada por el Decreto 103/1998 (BOPV, n 119, 26-6-98) y está formada por doce representantes del movimiento asociativo (cuatro por cada territorio) y cuatro representantes del propio instituto.

11. El Foro para la Igualdad entre Hombres y Mujeres y la Red de Entidades Parekatuz en Bizkaia y la Comisión de Igualdad de Oportunidades en Araba. 
Tabla 8. Mujeres y hombres en las ejecutivas de los partidos de la CAPV, 2002

\begin{tabular}{|l|r|r|r|r|c|}
\hline & Total & \multicolumn{2}{|c|}{ Mujeres } & \multicolumn{2}{c|}{ Hombres } \\
\hline & & Abs. & $\%$ & Abs. & $\%$ \\
\hline PNV & 14 & 1 & 7,1 & 13 & 92,9 \\
\hline EA & 12 & 5 & 41,7 & 7 & 58,3 \\
\hline BATASUNA & 32 & 9 & 28,1 & 23 & 71,9 \\
\hline PSE-EE & 22 & 10 & 45,5 & 12 & 54,5 \\
\hline IU-EB & 14 & 5 & 35,7 & 9 & 64,3 \\
\hline PP & 60 & 23 & 38,3 & 37 & 61,7 \\
\hline UA & 3 & 1 & 33,3 & 2 & 66,7 \\
\hline
\end{tabular}

Fuente: Emakunde: Cifras sobre la Situación de Mujeres y Hombres en Euskadi 2002.

Tabla 9. Mujeres y hombres en las ejecutivas de los principales sindicatos de la CAPV, 2002

\begin{tabular}{|l|c|c|c|c|c|}
\hline & Total & \multicolumn{2}{|c|}{ Mujeres } & \multicolumn{2}{c|}{ Hombres } \\
\hline & & Abs. & $\%$ & Abs. & $\%$ \\
\hline ELA & 12 & 3 & 25,0 & 9 & 75,0 \\
\hline CC.OO. & 24 & 4 & 16,7 & 20 & 83,3 \\
\hline LAB & 12 & 3 & 25,0 & 9 & 75,0 \\
\hline UGT & 9 & 2 & 22,2 & 7 & 77,8 \\
\hline
\end{tabular}

Fuente: Emakunde: Cifras sobre la Situación de Mujeres y Hombres en Euskadi 2002.

elevados corresponden a ELA y a LAB, en los que las mujeres constituyen la cuarta parte de la dirección.

El asociacionismo es un importante cauce para la participación social y política de la ciudadanía. El mundo asociativo constituye en la actualidad una realidad diversa y plural compuesta por grupos muy diferentes. Los índices de pertenencia a estos grupos y asociaciones en el caso de la CAPV muestran, una vez más, que los hombres participan en mayor medida que las mujeres en todos los tipos, destacando principalmente las sociedades deportivas, los partidos políticos y los sindicatos.

El total de asociaciones registradas en la CAPV es 12.293; de ellas, sólo 277 son asociaciones de mujeres, es decir, suponen el 2,25\% sobre el conjunto. Considerando los objetivos a los que responden estas asociaciones, la inmensa mayoría de ellas, 224 (el 80,9\% de las asociaciones de mujeres), se ubican en la categoría de "promoción de la mujer», es decir, buscan la mejora de calidad de vida de las mujeres. La siguiente categoría corresponde a los grupos feministas, 36 , que suponen el $13 \%$ sobre e total de asociaciones de mujeres. 
Tabla 10. Porcentajes de pertenencia a sociedades y asociaciones según sexo

\begin{tabular}{|l|c|c|c|}
\hline & Total & Hombres & Mujeres \\
\hline Sociedades Deportivas & 15,2 & 21,9 & 9,0 \\
\hline Sociedades Ecologistas & 1,0 & 1,2 & 0,8 \\
\hline Sociedades Gastronómicas & 3,3 & 5,7 & 1,1 \\
\hline Sociedades Culturales & 5,9 & 6,4 & 5,4 \\
\hline Asociaciones de Mujeres & 1,2 & 0,1 & 2,2 \\
\hline Asociaciones Profesionales & 3,4 & 4,1 & 2,7 \\
\hline Partidos Políticos/Sindicatos & 6,9 & 10,6 & 3,4 \\
\hline Otros colectivos & 6,6 & 5,8 & 7,3 \\
\hline
\end{tabular}

Fuente: Eustat, Encuesta de Condiciones de vida, 1999.

Población de 16 y más años. Porcentajes verticales.

Tabla 11. Asociaciones de mujeres registradas por tipo de asociación, 1999

\begin{tabular}{|l|r|r|r|c|}
\hline & Total & Araba & Bizkaia & Gipuzkoa \\
\hline Promoción de la mujer & 224 & 44 & 111 & 69 \\
\hline Grupos feministas & 36 & 8 & 16 & 12 \\
\hline Madres solteras & 3 & & 3 & \\
\hline Amas de casa & 14 & 3 & 10 & 1 \\
\hline Total asociaciones de mujeres & 277 & 55 & 140 & 82 \\
\hline Total asociaciones de todo tipo & 12.293 & 2.081 & 6.123 & 4.089 \\
\hline
\end{tabular}

Fuente: Emakunde: Cifras sobre la Situación de Mujeres y Hombres en Euskadi 2002.

\section{PARTICIPACIÓN POLÍTICA AUTÓNOMA}

El apartado sobre la participación política autónoma hace referencia, por un lado, a dos de los principales indicadores para expresar las actitudes generales respecto a la política: el interés hacia la política y la frecuencia con las que se habla de política; por otro lado, incluye datos acerca de las principales formas concretas de participación política en función del sexo. El interés por la política de la ciudadanía de la CAPV es relativamente elevado puesto que casi un tercio, el 31,8\%, afirma tener mucho o bastante interés por la materia. Existen algunas diferencias en función del sexo en el sentido de que las mujeres manifiestan un menor interés al de los hombres: la suma de quienes muestran mucho y bastante interés en las mujeres es de $29 \%$ mientras que para los hombres es del $34,7 \%$. Otra forma muy utilizada para expresar la valoración hacia la política consiste en medir la frecuencia con la que se habla del tema. De acuerdo con este indicador se vuelve a hacer patente, de manera aún más acusada, la diferencia por sexos puesto que el 40,7\% de las mujeres no habla nunca de política frente al 28,2\% de los hombres que señala la misma respuesta. 
Tabla 12. Interés por la política (porcentajes verticales)

\begin{tabular}{|l|c|c|c|}
\hline & \% Total & \% Mujeres & \% Hombres \\
\hline Mucho & 6,3 & 5,6 & 7,0 \\
\hline Bastante & 25,5 & 23,4 & 27,7 \\
\hline Poco & 38,0 & 38,5 & 37,6 \\
\hline Nada & 29,7 & 31,8 & 27,5 \\
\hline NS/NC & 0,5 & 0,7 & 0,2 \\
\hline
\end{tabular}

Fuente: Euskobarometro, mayo 2003.

Tabla 13. Frecuencia con la que habla de política (porcentajes verticales)

\begin{tabular}{|l|c|c|c|}
\hline & \% Total & \% Hombres & \% Mujeres \\
\hline Frecuentemente & 10,6 & 13,2 & 8,1 \\
\hline De vez en cuando & 54,4 & 58 & 50,9 \\
\hline Nunca & 34,5 & 28,2 & 40,7 \\
\hline NS/NC & 0,5 & 0,6 & 0,3 \\
\hline
\end{tabular}

Fuente: Universidad de Deusto: Los valores en la Comunidad Autónoma de Euskadi y Navarra, Bilbao, Universidad de Deusto, 2000. Emakunde: Cifras sobre la Situación de Mujeres y Hombres en Euskadi 2001.

Los datos incluyen la CAPV y la Comunidad Foral de Navarra.

La medición de las formas concretas de participación política muestra, en todos los casos, menores índices para las mujeres. Si tenemos en cuenta las diferentes modalidades, incluso en las actividades legales más usuales (como participar en manifestaciones y firmar peticiones) las mujeres presentan porcentajes inferiores de actividad. Por otra parte, en las acciones ilegales (como participar en huelgas no autorizadas, secundar boicots u ocupar edificios) los porcentajes de los hombres suelen ser muy superiores a los de las mujeres.

Tabla 14. Formas de participación política (porcentajes verticales)

\begin{tabular}{|l|c|c|c|}
\hline & Total & Hombres & Mujeres \\
\hline Participar en manifestaciones autorizadas & 51,4 & 57,1 & 46,0 \\
\hline Firmar una petición & 44,9 & 48,2 & 41,8 \\
\hline Participar en huelgas no autorizadas & 17,3 & 23,6 & 11,2 \\
\hline Secundar boicots & 12,9 & 16,7 & 9,3 \\
\hline Ocupar edificios o fábricas & 3,1 & 4,9 & 1,3 \\
\hline
\end{tabular}

Fuente: Universidad de Deusto: Los valores en la Comunidad Autónoma de Euskadi y Navarra. Bilbao, Universidad de Deusto, 2000. Emakunde: Cifras sobre la Situación de Mujeres y Hombres en Euskadi 2001.

Los datos incluyen la CAPV y la Comunidad Foral de Navarra. 


\section{CONCLUSIONES}

Los datos recogidos a lo largo de este artículo confirman las dos tendencias generalizadas en los últimos tiempos respecto a la participación política de las mujeres: a pesar del evidente aumento en su acceso a las instituciones y organizaciones políticas aún sigue siendo patente el desequilibrio en el protagonismo de hombres y mujeres tanto en la presencia en los poderes públicos como en los cauces fundamentales para la participación. Pero jes esto verdaderamente importante? ¿qué significado tiene la participación política en los sistemas políticos actuales? La respuesta a estas preguntas requiere reflexionar en torno a dos dimensiones científicas distintas: por un lado, las principales aportaciones empíricas respecto a los factores que explican la menor actividad política de las mujeres y, por otro lado, el abundante debate teórico sobre la democracia representativa y la democracia participativa.

Desde la perspectiva empírica, la principal idea que subrayan los estudios es la pervivencia en la desigualdad en el reparto de recursos políticos: en general, las mujeres carecen de las características, atributos y medios que configuran el perfil de los participantes en política. Siendo esto destacable, aún resulta más llamativo que incluso cuando poseen este tipo de recursos existen diferencias notables en la capacidad para convertirlos en actividad política. Es decir, la disposición de los mismos medios y atributos no se traducen en actividad política de la misma manera en los hombres y en las mujeres ${ }^{12}$. Aunque avancemos hacia un reparto más equitativo de los recursos y cada vez haya más mujeres con perfil de participantes, esto no garantiza el aumento de su participación política. Esta mayor inhibición o apatía política se presenta así como un problema complejo que no va atenuarse progresiva e inexorablemente a medida que las mujeres accedan a esferas que les estaban vedadas con anterioridad.

En lo que respecta al debate teórico planteado entre las distintas perspectivas democráticas, podemos resumir, de modo general y simple, que la democracia de representación se materializa a través de mecanismos de elección mientras que la democracia participativa se refleja en la creación de cauces para la deliberación y la decisión. Precisamente las propuestas prácticas de la democracia participativa surgen como respuesta a las insuficiencias de la democracia representativa, subrayando la exigencia de que las personas no deleguen en otras la decisión sobre todos los asuntos que les conciernen, es decir, que en parte sean colectiva y participativamente protagonistas más o menos directos de la resolución de sus intereses y demandas. Los partidarios de la democracia participativa abogan por el establecimiento paulatino de marcos jurídicos y políticos que posibiliten y potencien ese ejercicio colectivo. ¿Qué consecuencias puede tener este debate en la reivindicación de una mayor presencia política de las mujeres? Durante los últimos años una parte importante del movimiento feminista ha subrayado las ventajas de la democracia participativa por su enorme potencial para la incorporación de la voz de las mujeres a las decisiones públicas. Sin

12. Verba, Sidney, Norman H. Nie y Jae-on Kim: Op. cit., p. 236. 
embargo, no podemos olvidar que una contraposición excesivamente tajante entre ambas perspectivas podría cuestionar la importancia de los instrumentos de representación, con lo que estaríamos contribuyendo a la creación de obstáculos para la legitimidad del acceso de las mujeres a los cauces tradicionales de la participación política.

La verdadera relevancia de la participación política de la mujeres consiste en considerarla un bien positivo en sí mismo en el sentido de que es un mecanismo de empoderamiento, es decir, aúna representación y participación. La reivindicación subyacente, expresada históricamente por el movimiento feminista, es que la diferencia sexual necesita reflejarse en los niveles institucionales de decisión y en los diversos cauces de participación política. La demanda de la representación sexual puede llegar a cuestionar las bases del funcionamiento de los sistemas políticos actuales, sustentado por lo general en la representación de características sociales entre las que no se suele incluir el sexo. Por eso, esta reivindicación supone un amplio proyecto político que va más allá de considerar a las mujeres como un mero grupo social y que pretende la transformación y profundización del propio sentido de la democracia. De acuerdo con este proyecto, las mujeres no son un grupo sino un sexo y «un sexo es una de las dos formas constitutivas del ser humano ${ }^{13}$. Desde esta perspectiva, lo verdaderamente relevante no es que las mujeres defiendan sus intereses como colectivo (cuya existencia es hasta discutible) sino que lo esencial es que esto se entienda como un instrumento para hacer relevante la dualidad de formas de la humanidad rompiendo con la consideración unívoca, supuestamente neutral e implícitamente masculina de los miembros de la sociedad.

\section{BIBLIOGRAFÍA}

Astelarra, Judith: Participación política de las mujeres, Madrid, CIS, 1990.

Barnes, Samuel y KaAse, Max: Political Action: Mass Participation in Five Western Democracies, Beverly Hills, Sage, 1979.

BeскwIтH, Karen: American women and political participation, Westport, Greenwood Press, 1986.

Castells, Carme: Perspectivas feministas en teoría política, Barcelona, Paidós, 1996.

Chapman, Jenny: Politics, Feminism and the Reformation of Gender, Londres, Routledge, 1993.

Christy, Carol A: "Trends in Sex Differences in Political Participation: A Comparative Perspective», en Marianne Githens, Pippa Norris, y Joni Lovenduski: Different Roles, Different Voices: Women and Politics in the United States and Europe, Nueva York, Harper Collins, 1994, pp. 27-36.

Cohen, Cathy J.; Kathleen B. Jones, y Joan C. Tronto: Women Transforming Politics, Nueva York, New York University Press, 1997.

Coole, Diana: Women in Political Theory, Hertfordshire, Harvester-Wheatsheaf, 1993.

Dalton, Russell J.: Citizen Politics, New Jersey, Chatham, 1996.

13. Mancina, Claudia: «Diferencia Sexual y Representación», Mientras Tanto, 48 (1992), pp. 51-58, cita de p. 52. 
De Miguel, Ana y Rosa Coвo: «Implicaciones políticas del feminismo», en Fernando Quesada (ed): Filosofía Política: Ideas políticas y movimientos sociales, Madrid, Trotta, 1997, pp. 203-215.

Duverger, Maurice: The Political Role of Women, Nueva York, UNESCO, 1955.

Easton, David y Jack Dennis: Children and the Political System, Chicago, Chicago University Press, 1980 (edición original de 1969).

Elizondo, Arantxa y Martínez Hernández, Eva: «Presencia de Mujeres y Política para la Igualdad entre los Sexos: el caso de las Instituciones Políticas Vascas (1980-1994)», Revista de Estudios Políticos, 89 (1995), pp. 345-368.

ELIzOndo, Arantxa: La presencia de las mujeres en los partidos politicos de la CAPV, Vitoria, Gobierno Vasco, 1999.

EMAKUnde: Cifras sobre la Situación de Mujeres y Hombres en Euskadi 2000.

- Cifras sobre la Situación de Mujeres y Hombres en Euskadi 2001.

- Cifras sobre la Situación de Mujeres y Hombres en Euskadi 2002.

Fishinin, James: Democracia y deliberación, Barcelona, Ariel, 1995.

Flora, Cornelia B. y Naomi B. Lynn: «Woman and Political Socialization: Considerations of the Impact of Motherhood», en Jane S. Jaquette (ed): Women in Politics, Nueva York, John Wiley, 1974, pp. 37-53.

Githens, Marianne: «The Elusive Paradigm. Gender, Politics and Political Behavior: The State of the Art», en Ada Finifter (ed): Political Science: The State of the Discipline, Washington D.C, APSA, 1983, pp. 471-499.

Hernes, Helga Ma: El Poder de las Mujeres y el Estado del Bienestar, Madrid, Vindicación Feminista, 1990.

IGLITZIN, Lynne: "The Making of the Apolitical Woman: Feminity and Sex-Stereotyping in Girls», en Jane S. Jaquette (ed): Women in Politics, Nueva York, John Wiley, 1974.

Inglehart, Ronald: El Cambio Cultural en las sociedades industriales avanzadas, Madrid, CIS, 1991.

Klingemann, Hans Dieter y Dieter Fuchs. Citizens and the State, Oxford, Oxford University Press, 1995.

LIPSET, Seymour M: Political Man, Londres, Heinemann, 1971.

Lovenduski, Joni y Pippa Norris: Gender and Party Politics, Londres, Sage, 1993.

Lovenduski, Joni: Women and European Politics, Brighton, Wheatsheaf, 1986.

Mancina, Claudia: «Diferencia Sexual y Representación», Mientras Tanto, 48 (1992), pp. 51-58.

Martínez Hernández, Eva: "Participación Ciudadana y Empoderamiento de las Mujeres: Los Consejos de Igualdad en la Comunidad Autónoma del País Vasco», Comunicación presentada en el VI Congreso de la AECPA, Barcelona, 2003.

Martínez Hernández, Eva y Arantxa Elizondo: «Women in Politics: are they really concerned about equality?», European Journal of Women Studies, 4-4 (1997), pp. 451-472.

Mclaverty, Peter: "The Limits to Public Participation in Capitalist Society: The Example of Britain", en Joan Font (ed): Public Participation and Local Governance, Barcelona, ICPS, 2003, pp. 33-50.

Milbrath, Lester W.: Political Participation, Chicago, Rand McNally, 1968.

Norris, Pippa: «Gender Differences in Political Participation in Britain: Traditional, Radical and Revisionist Models», Government and Opposition, 26-1 (1991), pp. 56-74.

- Critical Citizens, Oxford, Oxford University Press, 1999.

Ortiz Corulla, Carmen: La participación política de las mujeres en la Democracia (19791986), Madrid, Instituto de la Mujer, 1987.

PHILliPs, Anne: Engendering Democracy, Cambridge, Polity Press\&Basil Blackwell, 1991. 
PINDADO, Fernando: La participación ciudadana en la vida de las ciudades, Barcelona, Ediciones del Serbal, 2000.

Pitkin, Hanna F.: The Concept of Representation, University of California Press, Berkeley, 1967.

Putnam, Robert D.: The Comparative Study of Political Elites, Englewood Cliffs, Nueva Jersey, Prentice Hall, 1976.

Randall, Vicky: Women and Politics: An International Perspective, Londres, MacMillan, 1987.

Rokkan, Stein: Citizens, Elections and Parties, Oslo, Universitatsforlaget, 1970.

SkJEIE, Hege: «The Rhetoric of Difference: on women's inclusion into political elites», Politics and Society, 19-2 (1991), pp. 233-263.

Uriarte, Edurne y Arantxa Elizondo: Mujeres en Política, Barcelona, Ariel, 1997.

Verba, Sidney, Norman H. NIE y Jae-on KIM: Participation and Political Equality: A SevenNation Comparison, Chicago, University of Chicago Press, 1978. 